\title{
The Vibration Study of DAMMAR Based Composite Bars by Using a New Euler-Bernoulli Theory
}

\author{
DUMITRU BOLCU ${ }^{1}$, MARIUS MARINEL STANESCU2*, ION CIUCA ${ }^{3}$, COSMIN MIHAI MIRITOIU ${ }^{1}$, ALEXANDRU BOLCU', \\ ROBERT CIOCOIU 3 \\ ${ }^{1}$ University of Craiova, Faculty of Mechanics, 165 Calea Bucuresti, 200620, Craiova, Romania \\ ${ }^{2}$ University of Craiova, Department of Applied Mathematics, 13 A.I.Cuza Str., 200396, Craiova, Romania \\ 3 Politehnica University Bucharest, Faculty of Engineering and Materials Science, 313 Splaiul Independentei, 060042, Bucharest, \\ Romania
}

In the paper, a new Euler-Bernoulli theory is presented, in which the bars eigenpulsations with rectangular section increase with the ratio between the bar width and thickness. This theory is experimentally verified for composite bars made of Dammar reinforced with cotton and flax plus one or two layers of fiber glass fabric. For the considered bars, we have experimentally determined the eigenfrequencies and the damping factor values. We have experimentally determined the Young modulus and breaking strength for the used resin and the obtained composites.

Keywords: composite bar, bar eigenpulsations, Euler-Bernoulli theory

The composite plates and bars can be analyzed through many theories which differ in particular by including or neglecting the effects of angular deformation, rotational inertia respectively. The elemental composite bars (ETB) and plates theory is based on the assumption that a straight line perpendicular on the medium surface before deformation, remais straight and normal on the medium surface during the deformation. It was found that, for laminates where the ratio between Young and shear modulus has values between 25-40, this theory overevaluates the structure natural frequencies.

Another theory known as the first shear deformation (FSDT), was made in [1] and developed later in [2]. This theory is based on a linear distribution and requires a corretion factor. In this theory, a straight line perpendicular on the medium plane before deformation remais straight, without keeping the perpendicularity on the medium plane during deformation. The results of some static and dynamic problems obtained in the FSDT hypotheses were concordantly with the exact solvings made with theory of elasticity and the experimental results. In the bars vibration case, this theory was firstly used by Timoshenko [3].

The exact theories of anisotropic plates and bars are based on a non-linear distribution of shear stresses on the thickness. The usage of high degree terms involves the including of extra unknown factors with mathematical difficulties for solving. At the third order theory, where the shear stresses distribution is parabolic, if the limitingconditions on the exterior surfaces are fulfilled, a correction factor is not necessary. Other high degree deformation theories are available in the engineering literature for static and dynamic beams analysis, in [5-7]. Later, in [8], a high degree trigonometrical theory is developed which cand fulfill all the limiting-conditions on the bars and plates exterior surfaces. Studied regarding the composite bars vibrations damping are made in [9-11].

In the last years, the interest of using natural fibers and resins for creating composite materials has increased. Natural fibers represent adequate reinforcing materials for composites because of the combination between good mechanical properties and advantages in the enviroment protection (regeneration and biodegradability). The usage of natural fibers as reinforcement has many advantages, such as: relatively low cost, abundance in nature, low weight, less damages to manufacturing equipments, good surface finishing for molded products (compared to the composites glass fiber based), good relative mechanical properties.

Various articles [12-19] present the properties of natural fibers and resins. In [20], the mechanical behaviour of some composite materials that have a Dammar based resin as matrix is studied. As reinforcement materials, fabrics of flax, hemp, cotton and silk. The main mechanical characteristics were determined for the used resin, but also for the composite materials obtained by using as reinforcements the mentioned fabrics. The possibilities of these materials usage combined with intelligent ones are presented in [21].

The weak compatibility of natural fibers with many polymeric matrices can lead to non-uniform fibers dispersions inside the matrix. In order to eliminate this disadvantage, the usage of some thermal rigid - biological matrices was tried (resins based on plants oil, soy-based resins or other vegetable oils) produced in a way to make the biodegradable. The natural resins are insoluble in water, but slightly soluble in oil, alcohool and partially in gas. The Turpentine, Rosin, Mastic are products resulted from pine resins distillation.

A study regarding their chemical composition is made in [22]. The vegetable resins are Sandarac, Copal and Dammar. From the fossils resins, the amber can be remembered and the Shellac, from the animal ones. A major disadvantage for these resins types is their high cost which makes them unapproachable even for large scale production. Other disadvantages for resins based on bio concept include brittleness, low temperature for hot deformation, high permeability at gases, an inadequate melting viscosity for a later manufacturing. All these disadvantages restrain their usage in a large area of applications [23]. 


\section{Theoretical background}

A bar with constant rectangular shape section is considered, with $b$ width and $h$ thickness (fig. 1).

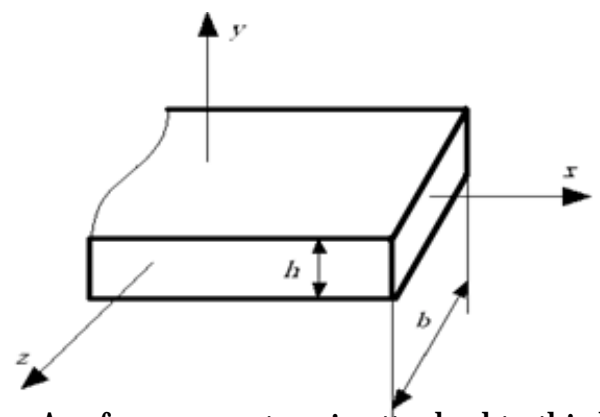

Fig. 1.

A reference system is attached to this bar, in this way: -the $x$ axis represents the longitudinal symmetry axis of the bar;

-the $y$ axis is focused on the bar thickness;

-the $z$ axis is focued on the width direction ( fig. 1).

During the vibrations, the bar points will have displacements along all three axes:

-displacement along the $x$ axis, marked as $u_{x}(x ; y ; z ; t)$;

-displacement along the y axis, marked as $u_{v}^{x}(x ; y ; z ; t)$; -displacement along the $z$ axis, marked a su $(x ; y ; z ; t)$.

If only the transversal vibrations are taken into account, neglecting the longitudinal and torque ones, the functions which characterizes the displacements over the three axes must fulfill the next conditions:

-the $u_{x}$ displacement must be an even function in the $z$ variable and uneven in y variable, so

$$
\begin{array}{r}
u_{x}(x ; y ;-z ; t)=u_{x}(x ; y ; z ; t), \\
u_{x}(x ;-y ; z ; t)=-u_{x}(x ; y ; z ; t) ;
\end{array}
$$

-the $u$ displacement must be an even function in both $z$ and y variables, so

$$
\begin{aligned}
& u_{y}(x, y ;-z ; t)=u_{y}(x, y ; z ; t) ; \\
& u_{y}(x ;-y ; z ; t)=u_{y}(x, y ; z ; t) ;
\end{aligned}
$$

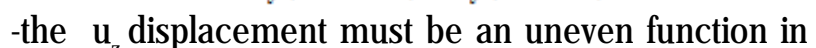
both $z$ and $y$ variables, so

$$
\begin{gathered}
u_{z}(x, y ;-z ; t)=-u_{z}(x ; y ; z ; t) ; \\
u_{z}(x ;-y ; z ; t)=u_{z}(x ; y ; z ; t) .
\end{gathered}
$$

By considering that on the exterior bar surface there are not exterior forces, the normal and shear stresses on these surfaces are zero. So, $z= \pm \frac{b}{2}$ and $y= \pm \frac{h}{2}$ for and we have:
$\quad$ for for $z= \pm \frac{b}{2}$

$$
\begin{aligned}
& \sigma_{x z}\left(x ; y ; \pm \frac{b}{2} ; t\right)=0 \\
& \sigma_{y z}\left(x ; y ; \pm \frac{b}{2} ; t\right)=0 \\
& \sigma_{z z}\left(x ; y ; \pm \frac{b}{2} ; t\right)=0
\end{aligned}
$$

-for for $y= \pm \frac{h}{2}$

$$
\begin{aligned}
& \sigma_{x y}\left(x \pm \frac{h}{2} ; z ; t\right)=0 \\
& \sigma_{y y}\left(x ; \pm \frac{h}{2} ; z ; t\right)=0 \\
& \sigma_{y z}\left(x ; \pm \frac{h}{2} ; z ; t\right)=0 .
\end{aligned}
$$

In order to simplify the calculus, the longitudinal deformations along an axis are considered to be made only by the normal stresses that correspond to that axis. The transversal contraction factors are neglected, similar like in the Euler-Bernoulli theory. In this case, we consider that the displacements have the next form

$$
\left\{\begin{array}{l}
u_{x}=\left(-y-\frac{2 z^{2}}{3 b} f(y)\right) \cdot \frac{\partial w(x, t)}{\partial x}, \\
u_{y}=\left(1-\frac{2 z^{2}}{3 b} f^{\prime}(y)\right) \cdot w(x ; t), \\
u_{z}=\left(\frac{2 z}{b}-\frac{8 z^{3}}{3 b^{3}}\right) f(y) \cdot w(x, t),
\end{array}\right.
$$

Where the function $\mathrm{f}(\mathrm{y})$ in uneven in the y variable and fulfills the conditions

$$
\begin{array}{r}
-f(y) \geq 0 \text { for any } y \geq 0, \\
f^{\prime}\left(\frac{h}{2}\right)=0, \\
f^{\prime \prime}\left(\frac{h}{2}\right)=0 .
\end{array}
$$

It is observed that for $z=0$, the displacements are the same with the ones from the Euler-Bernoulli theory. The deformations tensor parts are:

$$
\begin{gathered}
\varepsilon_{x x}=\left(-y-\frac{2 z^{2}}{3 b} f(y)\right) \cdot \frac{\partial^{2} w(x, t)}{\partial x^{2}}, \\
\varepsilon_{y y}=-\frac{2 z^{2}}{3 b} f^{\prime}(y) \cdot w(x, t), \\
\varepsilon_{z z}=\left(\frac{2}{b}-\frac{8 z^{2}}{3 b^{2}}\right) f(y) \cdot w(x, t) \\
\gamma_{x y}=-\frac{4 z^{2}}{3 b} f^{\prime}(y) \cdot w(x, t), \\
\gamma_{x z}=\frac{2 z}{3 b}\left(1-\frac{4 z^{2}}{b^{2}}\right) f(y) \cdot \frac{\partial w(x, t)}{\partial x}, \\
\gamma_{y z}=\frac{2 z}{3 b}\left(1-\frac{4 z^{2}}{b^{2}}\right) f^{\prime}(y) \cdot w(x, t) .
\end{gathered}
$$

In the Euler-Bernoulli theory, the transversal vibrations equation is:

$$
\iint_{(S)} \ddot{u} \rho d S+\frac{\partial^{2} M}{\partial x^{2}}=p_{y}
$$

where

$\rho$ - is the density;

$\mathrm{p}_{\mathrm{v}}$ - is the external loading which acts upon the bar length;

$\mathrm{M}$ - is the bending moment that is determined with the relation

$$
M=-\iint_{(S)} y \sigma_{x x} d S=-\iint_{(S)} y E \varepsilon_{x x} d S,
$$

where $\mathrm{E}$ is the Young modulus.

If a Taylor expansion in series in considered for $f(y)$ function, the terms up to seventh power are kept, in the (7) conditions, it has the form:

$$
f(y)=k\left(\frac{y^{3}}{h^{3}}-\frac{24 y^{5}}{5 h^{5}}+\frac{48 y^{7}}{7 h^{7}}\right),
$$


where $k$ is a positive constant. With this choice of $f(y)$ function, the stresses and displacements conditions in the medium layer are identical with the ones from EulerBernoulli classical theory for the bars vibrations study.

The motion equation (9) has the form:

$$
\rho b h\left(1-\frac{k}{315} \frac{b}{h}\right) \ddot{w}(x ; t)+E \frac{b h^{3}}{12}\left(1+\frac{k}{315} \frac{b}{h}\right) \frac{\partial^{4} w(x, t)}{\partial x^{4}}=p_{y}
$$

Inserting

$$
\begin{aligned}
& A=b h\left(1-\frac{k}{315} \frac{b}{h}\right), \\
& I=\frac{b h^{3}}{12}\left(1+\frac{k}{315} \frac{b}{h}\right) .
\end{aligned}
$$

the equation (12) becomes

$$
\rho A \ddot{w}(x, t)+E I \frac{\partial^{4} w(x, t)}{\partial x^{4}}=p_{y},
$$

The equation (15) is identical with the classical equation Euler-Bernoulli for bars vibration study. The bar free vibrations have the next form:

$$
w(x, t)=\sum_{n} W_{n}(x) \sin \left(\omega_{n} t+\varphi_{n}\right)
$$

where the $W_{n}(x)$ are the eigenfunctions and depend on the bar limiting-conditions.

The eigenpulsations are determined with the relation:

$$
\omega_{n}=\frac{\beta_{n}^{2}}{l^{2}} \sqrt{\frac{E I}{\rho A}}
$$

where $\beta$ depend on the bar limiting-conditions.

With (13) and (14), the eigenpulsations are:

$$
\omega_{n}=\frac{\beta_{n}^{2} h}{2 \sqrt{3} l^{2}} \sqrt{\frac{E}{\rho}} \sqrt{\frac{1+\frac{k}{315} \frac{b}{h}}{1-\frac{k}{315} \frac{b}{h}}}
$$

For $\mathrm{k}=0$, the eigenpulsations given by (18) are the same with the eigenpulsations given by the classical EulerBernoulli theory for the bars free vibrations with rectangular section. If $k$ is different from zero, the eigen pulsations values increase with the bar width, more precisely with the ratio between the bar width and thickness.

Actually, because of the internal frictions and air interaction, all the vibrations are damped. The presence of energy dissipation mechanisms is now accepted in all the models used for simulation of mechanical vibrations in mechanical systems. The study of damping phenomenon is presented in [24], by inserting some terms, having the form $2 c_{0} \dot{w}$ or $-2 c_{1} \frac{\partial^{2} w}{\partial x^{2}}$ or $2 c_{2} \frac{\partial^{4} \dot{w}}{\partial x^{4}}$, in the motion equation. The term $2 c_{0} \dot{w}$ inserts the so-called external or viscous damping. The aplitude of all the vibration modes (modal amplitudes) are damped at the same rate, contrary to the experiment. A natural interpretation of the $-2 c_{1} \frac{\partial^{2} \dot{w}}{\partial x^{2}}$ term is that the damping force is proportional with the bending rate. The presence of $2 c_{2} \frac{\partial^{4} \dot{w}}{\partial x^{4}}$ term means that the damping rates of vibration eigenmodes depend proportionally on the frequency sqare value. This is the socalled Kelvin-Voigt model of internal damping.

In the presence of damping, the bars free vibrations have the form:

$$
w(x, t)=\sum_{n} W_{n}(x) e^{-\mu_{n} t} \sin \left(\sqrt{\omega_{n}^{2}-\mu_{n}^{2}} t+\varphi_{n}\right)
$$

where $\mu_{n}$ is the damping factor for the vibration $n$ mode. The term $2 c_{0} \dot{w}$ leads to a constant damping factor, the term $-2 c_{1} \frac{\partial^{2} \dot{w}}{\partial x^{2}}$ leads to a damping factor in inverse proportion with the bar length sqare value, and in the case of $2 c_{2} \frac{\partial^{4} \dot{w}}{\partial x^{4}}$ term presence the damping factor is in inverse proportion with the fourth power of bar length.

\section{Experimental measurements}

The mechanical properties of composite materials reinforced with natural fibers can be very different because of the fibers properties variation. Even in the engineering literature there are differences of assesments. These reasons show that, in the case of new composite materials, it is necessary to experimentally determine the mechanical properties.

We have made samples based on the natural Dammar resin. The composite materials based only on this resin have a very long hardening time. In order to remove this deficiency, we have used a small quantity of synthetic resin. More precisely, we have used 75\% Dammar and $25 \%$ synthetic resin. The obtained sample sets had densities between $1.05-1.07 \mathrm{~g} / \mathrm{cm}^{3}$.

The samples were tensile tested to determine the mechanical characteristics. An universal testing machine for static and dynamic tests was used, with the maximum loading of $300 \mathrm{kN}$ - Walter Bai. The tensile test was made according to the stipulations from SR EN ISO 6892-1:2010. The main mechanical characteristics obtained for the combination Dammar - epoxy resin are:

- breaking strength between 21-24 MPa;

- breaking elongation between 1.98-2.64\%;

- transversal contraction factor between 0.47-0.56;

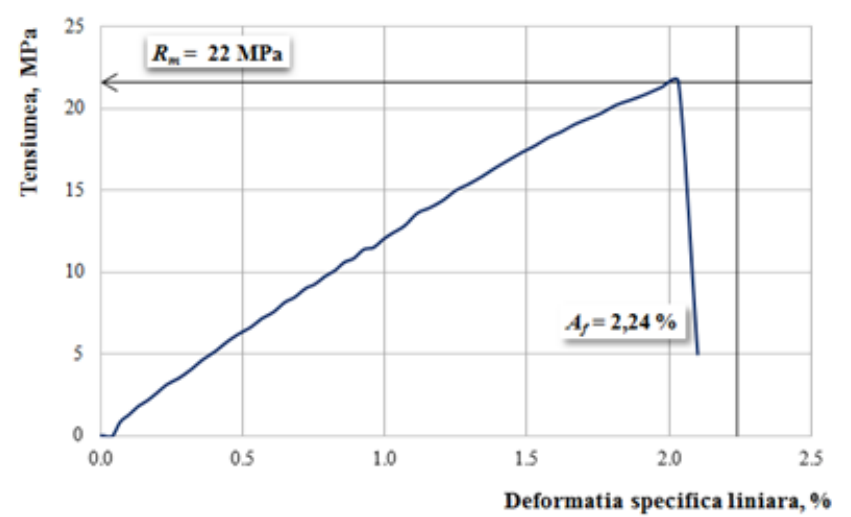

Fig. 2. Characteristic curve for a Dammar resin based sample

- elasticity modulus between 1189-1335 MPa.

In the figure 2, the characteristic curve stress - strain for a representative sample made from this resin is presented.

We have made a first samples set from this combined resin reinforced with:

-flax fabric, with the speciffic mass of $250 \mathrm{~g} / \mathrm{m}^{2}$. We have used 12 layers, the obtained composite having the resin mass fraction of 0.56 and the density of $1.15 \mathrm{~g} / \mathrm{cm}^{3}$.

-cotton fabric, with the speciffic mass of $130 \mathrm{~g} / \mathrm{m}^{2}$. We have used 12 layers, the obtained composite having the resin mass fraction of 0.58 and the density of $1.11250 \mathrm{~g} /$ $\mathrm{cm}^{3}$. 


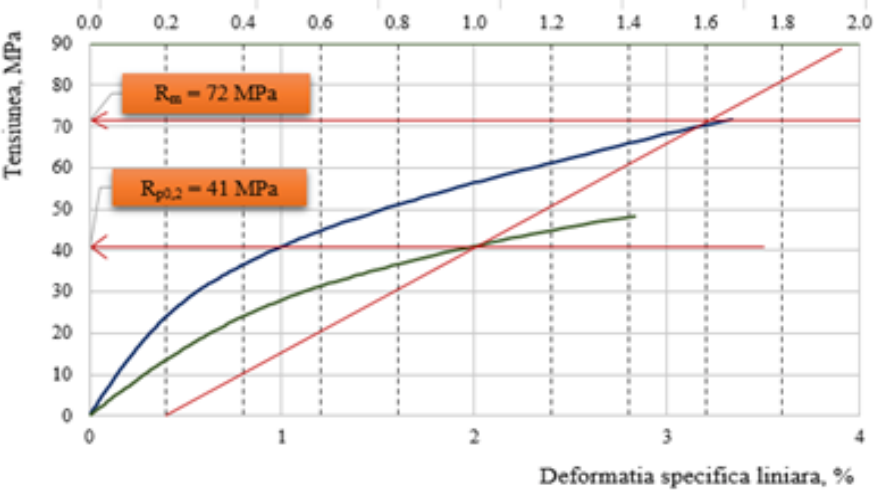

Fig. 3. The characteristic curve for a sample from Dammar based resin reinforced with flax fiber

Samples from this set were tensile tested. In the figure 3 , the characteristic curve stress - strain, for a representative sample made from Dammar based resin reinforced with flax fabric, is presented.

The main mechanical characteristics obtained for the Dammar resin based samples, reinforced with flax fiber are:

-breaking strength between 71-74 MPa;

-breaking elongation between 3.2-3.5 \%;

-transversal contraction factor between 0.33-0.37;

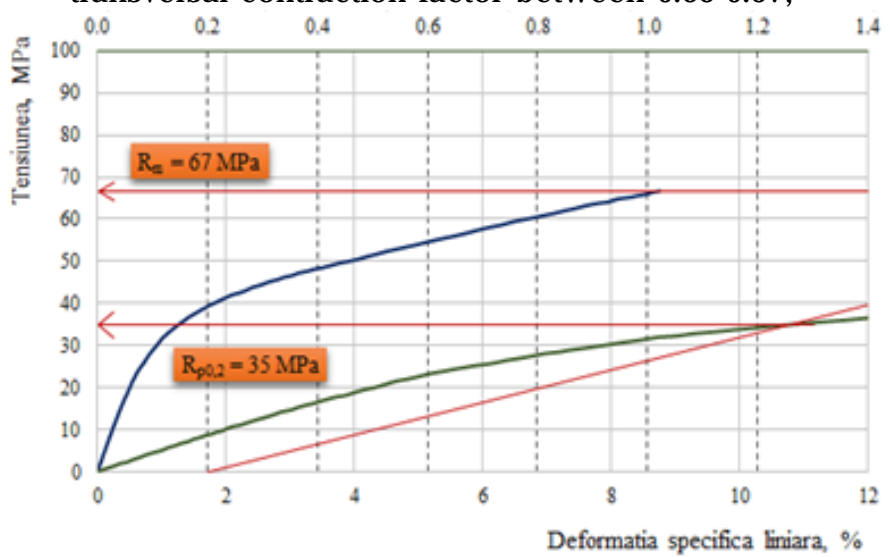

Fig. 4. The characteristic curve for a sample from Dammar based resin reinforced with cotton fiber

-modulus of elasticity between 5072-5215 MPa.

In the figure 4, the characteristic curve stress - strain for a representative sample made from Dammar based resin reinforced with cotton fabric, is presented.

The main mechanical characteristics obtained for the Dammar resin based samples, reinforced with cotton fiber are:

-breaking strength between 63-67 MPa;

-breaking elongation betw een 8.4-8.8\%;

-transversal contraction factor between 0.23-0.27;

-modulus of elasticity between 3297-3415 MPa.

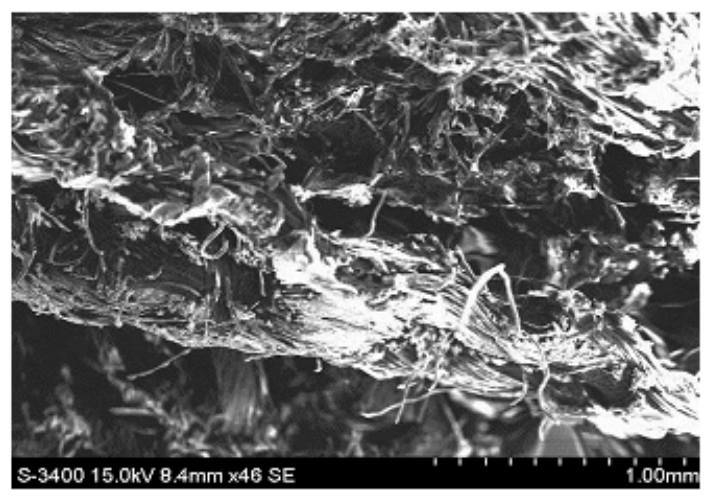

Fig. 5. An image from the breaking section for a sample reinforced with cotton

In the figure 5 an image from the breaking section for a sample reinforced with cotton is presented.

We have experimentally determined the damping factor for these samples sets. The studied samples had the length of $200 \mathrm{~mm}$ and widths of $10 \mathrm{~mm}, 20 \mathrm{~mm}, 30 \mathrm{~mm}$ and were clamped at one end, and the vibration measurement was made in the free end. The free length for each studied bar was 100, 120, 140 and $160 \mathrm{~mm}$.

The used measuring apparatus was:

-accelerometer with the $0.04 \mathrm{pC} / \mathrm{ms}^{-2}$ sensitivity;

-data acquisition system SPIDER 8;

-signal conditioner NEXUS 2692-A-014 connected to the SPIDER 8 system.

The data acquisition set was made with the CATMAN EASY software, and the frequency measuring field was between $0-2.400 \mathrm{~Hz}$ from SPIDER 8.

In the figure 6, the vibration experimental recording for the sample from the first set reinforced with flax, with the width of $10 \mathrm{~mm}$ and the free length of $170 \mathrm{~mm}$, is presented.

In the figure 7, the method of determining the damping factor for the figure 6 recording is presented. The damping factor per unit mass was determined with the relation:

$$
\mu=\frac{1}{t_{2}-t_{1}} \ln \frac{w_{1}}{w_{2}}
$$

- $t_{1}$ and $t_{2}$ are the time values where are obtained two maximum assests of the experimentally recorded diagram;

- $w_{1}$ is the maximum value at the $t_{1}$ moment of time and $w_{2}$ is the minimum value at the $t$ moment of time.

In the table 1, the values experimentally determined for the damping factor and the first vibration mode frequency, are presented for the samples reinforced with flax.

In the table 2, the values experimentally determined for the damping factor and the first vibration mode frequency, are presented for the samples reinforced with cotton.

We have made a second samples set from this combined resin reinforced with:

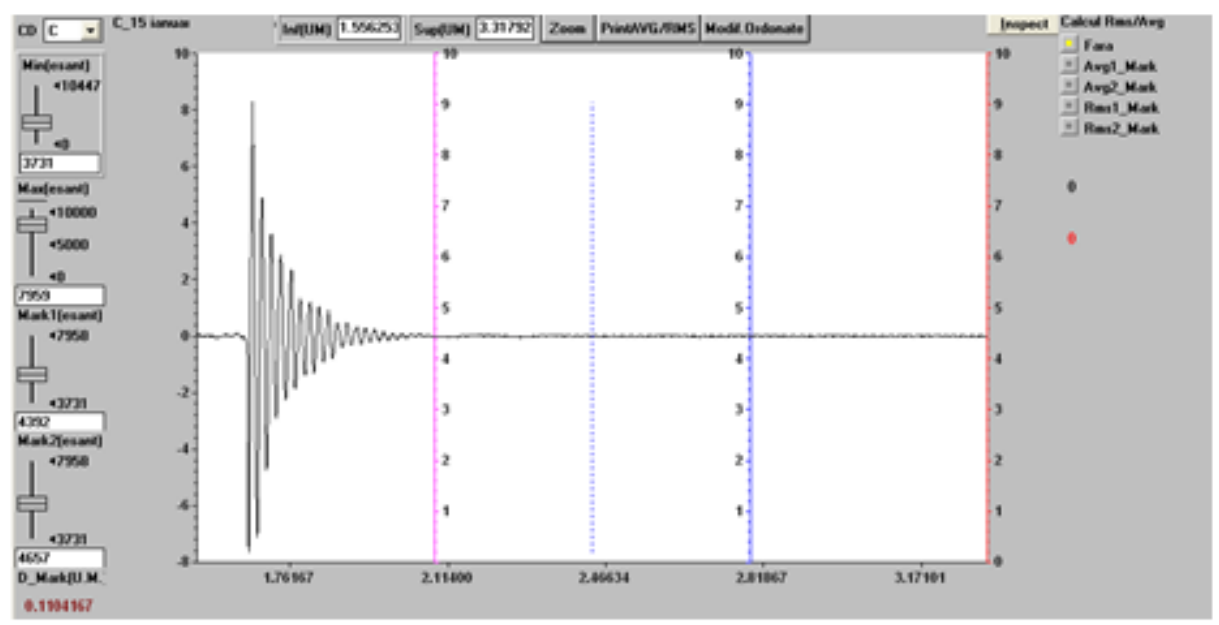

Fig. 6. Vibration experimental recording for the sample reinforced with flax, $10 \mathrm{~mm}$ width and $170 \mathrm{~mm}$ free length 


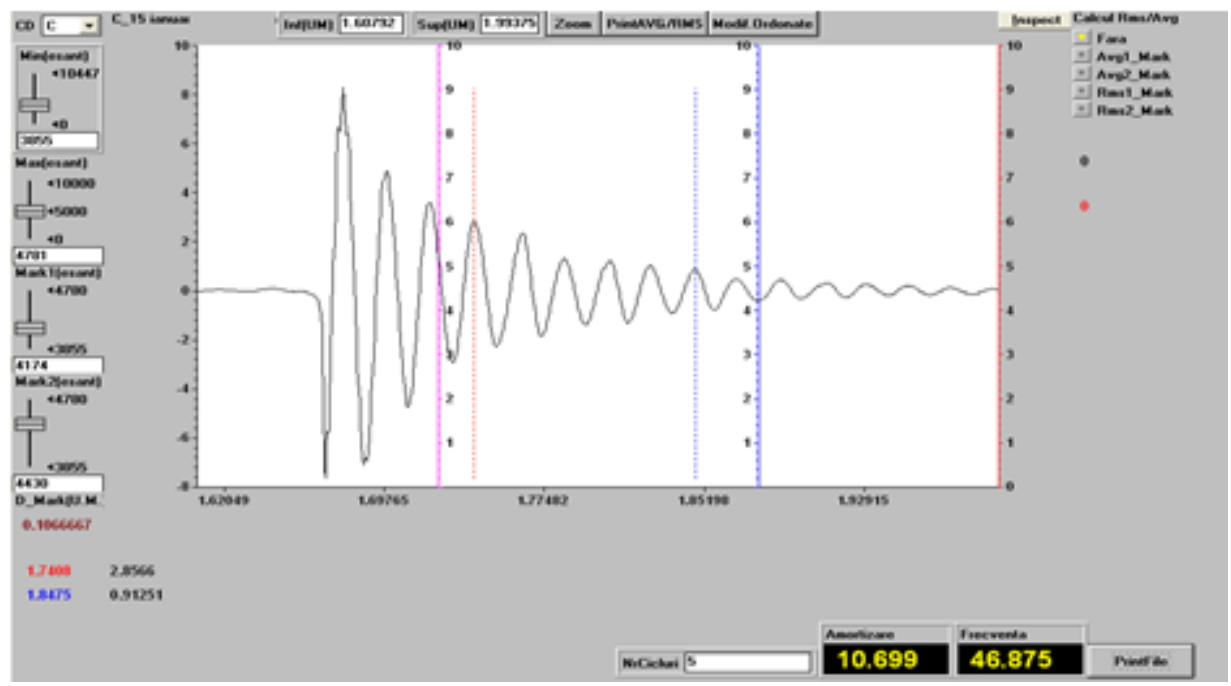

Fig. 7. Damping factor determination for the sample reinforced with flax, $10 \mathrm{~mm}$ width and $170 \mathrm{~mm}$ free length

- 6 layers of flax fabric, having in the middle a layer of glass fiber;

- 12 layers of cotton fabric, having in the middle a layer of glass fiber.

In the table 3, the values experimentally determined for the damping factor and the first vibration mode frequency, are presented for the second set of samples reinforced with flax.
In the table 4, the values experimentally determined for the damping factor and the first vibration mode frequency, are presented for the second set of samples reinforced with cotton.

We have made the third samples set from this combined resin reinforced with:

- 6 layers of flax fabric, having in the middle two layers of glass fiber,

\begin{tabular}{|c|c|c|c|c|c|c|}
\hline \multirow{2}{*}{ Width } & \multicolumn{2}{|c|}{$10 \mathrm{~mm}$} & \multicolumn{2}{c|}{$20 \mathrm{~mm}$} & \multicolumn{2}{c|}{$30 \mathrm{~mm}$} \\
\cline { 2 - 7 } & $\begin{array}{c}\text { Damping } \\
\text { factor } \\
{\left[\mathrm{s}^{-1}\right]}\end{array}$ & $\begin{array}{c}\text { Frequency } \\
{[\mathrm{Hz}]}\end{array}$ & $\begin{array}{c}\text { Damping } \\
\text { factor } \\
{\left[\mathrm{s}^{-1}\right]}\end{array}$ & $\begin{array}{c}\text { Frequency } \\
{[\mathrm{Hz}]}\end{array}$ & $\begin{array}{c}\text { Damping } \\
\text { factor } \\
{\left[\mathrm{s}^{-1}\right]}\end{array}$ & $\begin{array}{c}\text { Frequency } \\
{[\mathrm{Hz}]}\end{array}$ \\
\hline 100 & 25.43 & 112.15 & 29.89 & 118.81 & 30.18 & 130.43 \\
\hline 120 & 18.87 & 81.63 & 22.42 & 87.59 & 21.37 & 93.02 \\
\hline 140 & 14.29 & 61.37 & 16.40 & 67.96 & 16.28 & 71.00 \\
\hline 160 & 10.55 & 46.07 & 11.10 & 50.42 & 11.22 & 53.81 \\
\hline
\end{tabular}

Table 1

THE DAMPING FACTOR AND FREQUENCY FOR THE SAMPLES REINFORCED WITH FLAX

\begin{tabular}{|c|c|c|c|c|c|c|}
\hline Width & \multicolumn{2}{|c|}{$10 \mathrm{~mm}$} & \multicolumn{2}{c|}{$20 \mathrm{~mm}$} & \multicolumn{2}{c|}{$30 \mathrm{~mm}$} \\
\cline { 2 - 7 } Length & $\begin{array}{c}\text { Damping } \\
\text { factor } \\
{\left[\mathrm{s}^{-1}\right]}\end{array}$ & $\begin{array}{c}\text { Frequency } \\
{[\mathrm{Hz}]}\end{array}$ & $\begin{array}{c}\text { Damping factor } \\
{\left[\mathrm{s}^{-1}\right]}\end{array}$ & $\begin{array}{c}\text { Frequency } \\
{[\mathrm{Hz}]}\end{array}$ & $\begin{array}{c}\text { Damping factor } \\
{\left[\mathrm{s}^{-1}\right]}\end{array}$ & $\begin{array}{c}\text { Frequency } \\
{[\mathrm{Hz}]}\end{array}$ \\
\hline 100 & 23.97 & 81.36 & 21.24 & 91.25 & 21.28 & 102.56 \\
\hline 120 & 15.76 & 58.25 & 16.99 & 65.03 & 16.03 & 73.39 \\
\hline 140 & 12.34 & 43.02 & 13.89 & 51.28 & 13.91 & 55.81 \\
\hline 160 & 9.54 & 34.04 & 9.74 & 40.60 & 9.58 & 42.40 \\
\hline
\end{tabular}

Table 2

THE DAMPING FACTOR AND FREQUENCY FOR THE SAMPLES REINFORCED WITH COTTON

Table 3

THE DAMPING FACTOR AND FREQUENCY FOR SAMPLES REINFORCED WITH FLAX AND A LAYER OF FIBER GLASS

\begin{tabular}{|c|c|c|c|c|c|c|}
\hline \multirow{2}{*}{ Width } & \multicolumn{2}{|c|}{$10 \mathrm{~mm}$} & \multicolumn{2}{c|}{$20 \mathrm{~mm}$} & \multicolumn{2}{c|}{$30 \mathrm{~mm}$} \\
\cline { 2 - 7 } & $\begin{array}{c}\text { Damping } \\
\text { factor } \\
{\left[\mathrm{s}^{-1}\right]}\end{array}$ & $\begin{array}{c}\text { Frequency } \\
{[\mathrm{Hz}]}\end{array}$ & $\begin{array}{c}\text { Damping } \\
\text { factor } \\
{\left[\mathrm{s}^{-1}\right]}\end{array}$ & $\begin{array}{c}\text { Frequency } \\
{[\mathrm{Hz}]}\end{array}$ & $\begin{array}{c}\text { Damping } \\
\text { factor } \\
{\left[\mathrm{s}^{-1}\right]}\end{array}$ & $\begin{array}{c}\text { Frequency } \\
{[\mathrm{Hz}]}\end{array}$ \\
\hline 100 & 17.27 & 77.86 & 17.72 & 88.16 & 17.91 & 100.42 \\
\hline 120 & 12.68 & 57.62 & 14.23 & 66.53 & 12.98 & 70.43 \\
\hline 140 & 11.27 & 43.40 & 11.58 & 50.20 & 10.12 & 55.52 \\
\hline 160 & 9.34 & 35.10 & 9.45 & 40.37 & 7.12 & 42.18 \\
\hline
\end{tabular}

Table 4

THE DAMPING FACTOR AND FREQUENCY FOR SAMPLES REINFORCED WITH COTTON AND A LAYER OF FIBER GLASS

\begin{tabular}{|c|c|c|c|c|c|c|}
\hline \multirow{2}{*}{ Width } & \multicolumn{2}{|c|}{$10 \mathrm{~mm}$} & \multicolumn{2}{c|}{$20 \mathrm{~mm}$} & \multicolumn{2}{c|}{$30 \mathrm{~mm}$} \\
\cline { 2 - 7 } & $\begin{array}{c}\text { Damping } \\
\text { factor } \\
{\left[\mathrm{s}^{-1}\right]}\end{array}$ & $\begin{array}{c}\text { Frequency } \\
{[\mathrm{Hz}]}\end{array}$ & $\begin{array}{c}\text { Damping } \\
\text { factor } \\
{\left[\mathrm{s}^{-1}\right]}\end{array}$ & $\begin{array}{c}\text { Frequency } \\
{[\mathrm{Hz}]}\end{array}$ & $\begin{array}{c}\text { Damping } \\
\text { factor } \\
{\left[\mathrm{s}^{-1}\right]}\end{array}$ & $\begin{array}{c}\text { Frequency } \\
{[\mathrm{Hz}]}\end{array}$ \\
\hline 100 & 14.79 & 66.39 & 13.10 & 75.16 & 15.78 & 85.33 \\
\hline 120 & 12.18 & 47.36 & 12.44 & 53.01 & 10.96 & 65.41 \\
\hline 140 & 9.87 & 36.44 & 9.62 & 41.27 & 9.70 & 47.03 \\
\hline 160 & 7.89 & 29.06 & 6.75 & 31.14 & 6.23 & 34.33 \\
\hline
\end{tabular}


Table 5

THE DAMPING FACTOR AND FREQUENCY FOR SAMPLES REINFORCED WITH FLAX AND TWO LAYERS OF FIBER GLASS

\begin{tabular}{|c|c|c|c|c|c|c|}
\hline Width & \multicolumn{2}{|c|}{$10 \mathrm{~mm}$} & \multicolumn{2}{c|}{$20 \mathrm{~mm}$} & \multicolumn{2}{c|}{$30 \mathrm{~mm}$} \\
\cline { 2 - 7 } Length & $\begin{array}{c}\text { Damping } \\
\text { factor } \\
{\left[\mathrm{s}^{-1}\right]}\end{array}$ & $\begin{array}{c}\text { Frequency } \\
{[\mathrm{Hz}]}\end{array}$ & $\begin{array}{c}\text { Damping } \\
\text { factor } \\
{\left[\mathrm{s}^{-1}\right]}\end{array}$ & $\begin{array}{c}\text { Frequency } \\
{[\mathrm{Hz}]}\end{array}$ & $\begin{array}{c}\text { Damping } \\
\text { factor } \\
{\left[\mathrm{s}^{-1}\right]}\end{array}$ & $\begin{array}{c}\text { Frequency } \\
{[\mathrm{Hz}]}\end{array}$ \\
\hline 100 & 19.75 & 110.20 & 20.66 & 125.16 & 18.36 & 139.94 \\
\hline 120 & 16.85 & 80.14 & 15.76 & 89.88 & 15.98 & 99.58 \\
\hline 140 & 12.02 & 61.67 & 13.35 & 66.99 & 13.46 & 72.89 \\
\hline 160 & 9.65 & 47.13 & 11.45 & 50.61 & 10.90 & 55.11 \\
\hline
\end{tabular}

Table 6

THE DAMPING FACTOR AND FREQUENCY FOR SAMPLES REINFORCED WITH COTTON AND TWO LAYERS OF FIBER GLASS

\begin{tabular}{|c|c|c|c|c|c|c|}
\hline \multirow{2}{*}{ Width } & \multicolumn{2}{|c|}{$10 \mathrm{~mm}$} & \multicolumn{2}{c|}{$20 \mathrm{~mm}$} & \multicolumn{2}{c|}{$30 \mathrm{~mm}$} \\
\cline { 2 - 7 } Length & $\begin{array}{c}\text { Damping factor } \\
{\left[\mathrm{s}^{-1}\right]}\end{array}$ & $\begin{array}{c}\text { Frequency } \\
{[\mathrm{Hz}]}\end{array}$ & $\begin{array}{c}\text { Damping } \\
\text { factor } \\
{\left[\mathrm{s}^{-1}\right]}\end{array}$ & $\begin{array}{c}\text { Frequency } \\
{[\mathrm{Hz}]}\end{array}$ & $\begin{array}{c}\text { Damping factor } \\
{\left[\mathrm{s}^{-1}\right]}\end{array}$ & $\begin{array}{c}\text { Frequency } \\
{[\mathrm{Hz}]}\end{array}$ \\
\hline 100 & 19.06 & 91.77 & 18.15 & 112.68 & 20.82 & 118.96 \\
\hline 120 & 15.61 & 60.95 & 13.08 & 79.93 & 14.15 & 84.95 \\
\hline 140 & 10.95 & 46.48 & 10.44 & 60.34 & 10.26 & 63.70 \\
\hline 160 & 8.47 & 39.72 & 7.26 & 46.65 & 8.32 & 46.04 \\
\hline
\end{tabular}

- 12 layers of cotton fabric, having in the middle two layers of glass fiber.

In the table 5, the values experimentally determined for the damping factor and the first vibration mode frequency, are presented for the third set of samples reinforced with flax.

In the table 6, the values experimentally determined for the damping factor and the first vibration mode frequency, are presented for the third set of samples reinforced with cotton.

\section{Conclusions}

The breaking sections and characteristics curves analysis shows that the breakage is suddenly made, although the ways for which the breakage appears can be different from a composite material to another. Therefore, a breakage type appears when the matrix is dettached from the fibers (which were plucked from the resin) and a breakage type that, with the fibers failure the matrix is broken too, keeping its contact with the fibers with the fibers in the place where the breakage took place (made by a perpendicular direction on the loading direction). The first breakage type apprard for the composites reinforced with cotton fibers which have high values of breaking elongation, and the second breakage type appeared at the composites with flax fibers that have low breaking elongation.

The characteristic curves analysis for the samples reinforced with cotton highlights the existance of three stages in the process of loading and deformation. In the first stage, a prportionality between the stress and strain exists, in which the Hooke law is verified. The second stage presents a non-linear character, in this stage there is the point where the yield stress appears for which residual strains of $0.2 \%$ appear after removing the loading. It is observed that the strain in this area is very close to the strain that appears at the resin breakage. In the third zone, the linear dependence between the stresses and strains reappears. In the first stage the loading is taken over by both the fibers longitudinally placed and the matrix which assures the composite material cohesion. In the second stage, the dependance stress - strain becomes non-linear because the breaking strength is obtained in the resin and it fails in certain points, there is lost the adhesion between the fibers and matrix, so fibers pluckings from the matrix appear. In the third stage, the stress-strain dependance becomes again linear. This fact suggests that the fabric fibers that are longitudinally disposed take over the whole loading, and the composite breakage is made when the breaking strength in the fibers is achieved.

It can be seen that the studied materials have very good damping vibrations properties. The damping factor has higher values for the samples reinforced with flax. By inserting one or two fiber glass layers leads to the damping factor decrease, for both samples reinforced with flax and cotton. The damping factor variation analysis show that its values are modified in inverse proportion with the bar length square value. This thing highlights that, from the three told damping mechanisms, the most prevelant is the one in which the damping force is porportional with the bending rate.

The elasticity moduli are proportional with the square values of vibration frequencies. The measured vibration frequencies show that the materials reinforced with flax have higher elasticity moduli than the ones reinforced with cotton. The same conclusion was obtained from the tensile tests results. This conclusion is also valid for the samples where an extra fiber glass was inserted.

The presented theory is based on an assymetric distribution of strains toward the medium plane, the area loaded to compression has a transversal expansion, and the area tensile loaded is straiten. As a consequence of this fact, there can be seen a free vibrations frequency increase, this phenomenon is also confirmed by the experimental results.

\section{References}

1.YANG, P.C., NORRIS, C.H., STAVSKY, Y., Elastic wave propagation in heterogeneous plates, International J ournal of Solids and Structures, 2(4), 1966, p. 665-684.

2.WHITNEY, J.M., PAGANO, N.Y.S., Shear deformation in heterogeneous anisotropic plates, J. Appl. Mech., 37(4), 1970, p. 1031-1036.

3.TIMOSHENKO, S.P., On the correction for shear of the differential equation for transverse vibrations of prismatic bars, Philosophical Magazine, 41(6), 1921, p. 742-746.

4.REDDY, J.N., Energy and variational methods in applied mechanics, John Wiley \& Sons, 1984. 
5.LIBRESCU, L., Formulation of an elastodynamic theory of laminated shear deformable flat panels, Journal of Sound and Vibration, 147(1), 1991, p. 1-12.

6.BHIMARADDI, A., CHANDRASHEKHARA, K., Observations on higherorder beam theory, J ournal of Aerospace Engineering Proceeding ASCE Technical Note, 6, 1993, p. 408-413.

7.KARAMA, M., AFAQ, K.S., MISTOU, S., Mechanical behaviour of laminated composite beam by new multi-layered laminated composite structures model with transverse shear stress continuity, International J ournal of Solids and Structures, 40, 2003, p. 1525-1546.

8.GHUGAL, Y.M., SHARMA, R., Hyperbolic shear deformation theory for flexure and vibration of thick isotropic beams, International J ournal of Computational Methods, 6(4), 2009, p. 585-604.

9.STANESCU, M.M., BOLCU, D., PASTRAMA, S.D., CIUCA, I., MANEA, I., BACIU, F., Determination of damping factor, to vibrations of composite beams, reinforced with carbon and Kevlar texture. Mat. Plast., 47, no. 4, 2010, p. 492

10.MIRITOIU, C.M., BOLCU, D., STANESCU, M.M., CIUCA, I., CORMOS, R., Determination of damping coefficients for sandwich bars with polypropylene honeycomb core and the exterior layers reinforced with metal fabric. Mat. Plast., 49, no. 2, 2012, p. 118

11.BURADA, C.O., MIRITOIU, C.M., BOLCU, D., STANESCU M.M., Experimental determinations of the damping factor and stiffness for new sandwich platbands with different reinforcement and core, Revista Românã de Materiale / Romanian Journal of Materials, 44(4), 2014, p. 405-413.

12.MOHANTY, A.K., MISRA, M., HINRICHSEN, G., Biofibers, biodegradable polymers and biocomposites: an overview, Macromol. Mater. Eng., 276-277, 2000, p. 1-24.

13.DHAKAL, H.N., ZHANG, Z.Y., RICARDSON, M.O.W., Effect of water absorption on the mechanical properties of hemp fibre reinforced unsaturated polyester composites, Compos. Sci. Technol., 67, 2007, p. 1674-1683.
14.AHMAD, I., BAHARUM, A., ABDULLAH, I., Effect of extrusion rate and fiber loading on mechanical properties of Twaron fiberthermoplastic natural rubber (TPNR) composites, J. Reinf. Plast. Compos., 25, 2006, p. 957-965.

15.KU, H., WANG, H., PATTARACHAIYAKOOP, N., TRADA, M., A review on the tensile properties of natural fibre reinforced polymer composite, Compos. Part B-Engineering, 42, 2011, p. 856-873.

16.KORONIS, G., SILVA, A., FONTUL, M., Green composites: A review of adequate materials for automotive apllications, Compos. Part BEngineering, 44, 2013, p. 120-127.

17.KABIR, M.M., WANG, H., LAU, K.T., CARDONA, F., Chemical treatments on plant-based natural fibre reinforced polimer composite: An overview, Compos. Part B-Engineering, 43, 2012, p. 2883-2892.

18.MEI-PO, H., WANG, H., JOONG-HEE, L., KIN-TAK, L., JINSONG, L., HUI, D., Critical factors on manufacturing processes of natural fibre composites, Compos. Part B-Engineering, 43, 2012, p. 3549-3562.

19.HOI-YAN, C., MEI-PO, H., KIN-TAK, L., CARDONA, F., HUI, D., Natural fibre-reinforced composites for bioengineering an enviromental engineering applications, Compos. Part B-Engineering, 40, 2009, p. 655-663.

20.STANESCU, M.M., Study regarding the mechanical behaviour of Dammar based composite materials, reinforced with natural fiber fabrics. Mat. Plast., 52, no. 4, 2015, p. 596

21.TARNITA, D., Wearable sensors used for human gait analysis, Rom J Morphol Embryo/ 2016, 57(2), pp 373-382.

22.PRATI, S., SCIUTTO, G., MAZZEO, R., TORRI, C., FABBRI, D., Application of ATR-far-infrared spectroscopy to the analysis of natural resins. Anal Bioanal Chem 2011;399:3081-91.

23.SUPRAKAS, SR., MOSTO, B., Biodegradable polymers and their layered silicate nanocomposites: in greening the 21st century materials world. Prog Mater Sci 2005;50:962-1079.

24. HERRMANN L., Vibration of the Euler-Bernoulli beam with allowance for dampings, Proceedings of the World Congress on Engineering 2008 Vol II ,WCE 2008, July 2 - 4, 2008, London, U.K.

Manuscript received: 18.08 .2016 